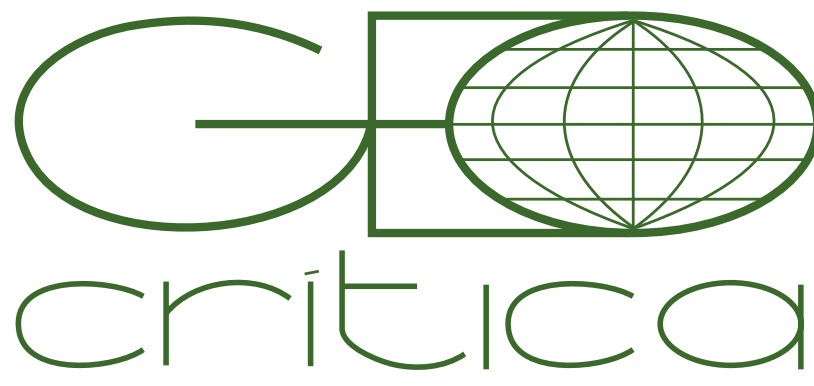

\section{Scripta Nova}

Revista Electrónica de Geografía y Ciencias Sociales Universidad de Barcelona

31 de enero de 2017

\title{
EL URBANISMO COMO ACTO DE COMUNICACIÓN: THEODORA KIMBALL HUBBARD Y LA BIBLIOTECA DE LA HARVARD LANDSCAPE ARCHITECTURE SCHOOL
}

\author{
María Cristina García González \\ Universidad Politécnica de Madrid \\ mariacristinagarciagonzalez@gmail.com
}

Salvador Guerrero

Universidad Politécnica de Madrid salvador.guerrero@upm.es

Recibido: 18 de septiembre de 2015. Aceptado:14 de abril de 2016

\begin{abstract}
Resumen. El urbanismo como acto de comunicación. Theodora Kimball Hubbard y la Biblioteca de la Harvard Landscape Architecture School

La bibliotecaria Theodora Kimball (1887-1935) contribuyó significativamente a la consolidación del urbanismo como nuevo ámbito disciplinar con el establecimiento de unos criterios de clasificación de la amalgama de conocimientos, conceptos, utillaje técnico y experiencias que surgieron en las tres primeras décadas del siglo XX. Se favorecía así la formación de una literatura específica que permitiera una mayor presencia del urbanismo tanto en los círculos académicos como en los ámbitos profesionales. Su trabajo, desarrollado en la Biblioteca de la Landscape Architecture School de la Universidad de Harvard, constituye uno de los principales nodos norteamericanos de las redes trasnacionales del urbanismo de su tiempo. Fue clave en ámbitos como la formación universitaria, la divulgación científica, la participación ciudadana, la irrupción del zoning como nueva herramienta de control del desarrollo urbano frente al city planning y el paso del landscape architecture al regional planning.
\end{abstract}

Palabras clave: Theodora Kimball Hubbard, paisajismo, urbanismo, zoning, regional planning.

\begin{abstract}
City Planning as an act of communication: Theodora Kimball Hubbard and the Harvard Landscape Architecture School Library

The librarian Theodora Kimball (1887-1935) was one of the key figures in the institutionalization of urban planning in the United States. She contributed to the advancement of professionalism of planning by establishing criteria to rationalize the amalgam of knowledge and experiences that were taking place, favouring the formation of a specific literature that would allow a greater presence in the academic and professional fields. Her work in the library of the Landscape School of Harvard University was one of the leading American node in the international network of knowledge through which flowed the key issues of urban debate period as technician training, dissemination of knowledge and citizen participation, the emergence of zoning as a tool for control of urban development versus positive nature of city planning and landscape architecture step from regional planning.
\end{abstract}

Keywords: Theodora Kimball Hubbard, landscape architecture, city planning, zoning, regional planning, 
If men define situations as real, they are real in their consequences.

Teorema de W. I. Thomas (1928)

El año 1909 puede considerarse una fecha de partida del urbanismo moderno. En el ámbito universitario se impartieron los primeros cursos dedicados al urbanismo: en Europa se creó el departamento de Civic Design, con la cátedra Level, en la Universidad de Liverpool (Inglaterra), bajo la dirección de Stanley Davenport Adshead; y en Norteamérica, se dictó el primer curso dedicado al urbanismo en la Harvard Landscape Architecture School, bajo la responsabilidad del arquitecto James Sturgis Pray (1871-1929).

En el ámbito de la difusión tuvo lugar la I National Conference on City Planning de Washington, primer congreso norteamericano dedicado íntegramente al urbanismo, a instancias del New York Committee on Congestion of Population, la asociación creada en 1907 bajo el liderazgo de reformador social Benjamin C. Marsh, con Florence Kelly, Charles Mulford Robinson, el abogado Edward Basset y George B. Ford entre otros. «¿Qué necesita el planeamiento americano?» fue el título de la ponencia de John Nolen (1869-1937). «Todo», se contestaba él mismo. Ese mismo año, una entidad privada, el Commercial Club de Chicago financió la publicación del report Plan of Chicago, correspondiente al plan de extensión de la ciudad redactado por Daniel H. Burnham y Edward H. Bennett en 1906. Además, se publicaron An Introduction to City Planning: Democracy's Challenge and the American City" de Benjamin Clark Marsh y Town Planning: Past, Present and Possible, del inglés Inigo Triggs.

En el campo de la legislación, se aprobó la ley inglesa Town and Country Planning Act, de la que tomaron nota iniciativas legales como la conocida Ley Cornudet de Francia (L'Aménagent, l'Embellissement et l'Extension des Villes, 1919) y el Estatuto Municipal español, de 1924, fecha de inicio del urbanismo municipalista. La ley inglesa tuvo como consecuencia directa dos significativos hechos: en primer lugar, se publicó el que con el tiempo se convertiría en el manual de urbanismo de referencia en Europa, Town planning in practice: an introduction of the art of designing cities and suburbs, del arquitecto inglés Raymond Unwin, planteado por su autor como una herramienta para la puesta en práctica de la ley, en cuya elaboración había participado activamente; en segundo lugar, se celebró en Londres el congreso organizado por el Royal Institute of British Architects ${ }^{1}$, que tuvo lugar en 1910, con la intención de dotar al urbanismo de un corpus teórico-práctico que permitiese su profesionalización. Este congreso reunió a arquitectos, paisajistas, ingenieros, sociólogos, abogados y políticos que venían trabajando en el urbanismo, como

1 Sobre las National Conference on City Planning véanse los proceedings editados al respecto. 
Daniel H. Burnham, G. L. Pepler, Charles Mulford Robinson, Josef Stübben, Rudolph Eberstadt, Eugène Hénard, Adolphe Augustin Rey, Thomas Hayton Mawson, Patrick Geddes, Raymond Unwin y Ebenezer Howard, entre otros, definiendo así el carácter multidisciplinar e trasnacional de la nueva ciencia.

En este contexto, llegó en 1911 a la Harvard Landscape Architecture School la joven bibliotecaria Theodora Kimball (1887-1935) con el cometido de organizar su biblioteca.

\section{La bibliotecaria de la Harvard Landscape Architecture School, 1913- 1924: Theodora Kimball Hubbard}

La Revista Municipal y de Intereses Económicos, de la que era director y propietario el catedrático de Gobierno Municipal y Urbanismo y director de la Escuela de Ciencias Políticas, Sociales y Económicas de la Universidad de La Habana, Francisco Carrera Jústiz (1857-1947), publicó en 1921 el que sería el primer artículo, en español, sobre el reconocimiento que merecía el trabajo desarrollado por Theodora Kimball, bibliotecaria de la Harvard Landscape Architecture School, en los diez años que llevaba al frente de dicha biblioteca.

Theodora Kimball había nacido en 1887 en West Newton, Massachussets, en el seno de una familia acomodada establecida en Boston por varias generaciones y no exenta de estímulos intelectuales. Mantuvo una relación muy estrecha con su único hermano, el arquitecto Sydney Fiske Kimball (1888-1955), que con el tiempo se convertiría en uno de los más reconocidos historiadores de la arquitectura norteamericana. Ambos recibieron una esmerada educación. Theodora asistió a la prestigiosa escuela Girl's Latin School de Boston, una de las primeras instituciones educativas femeninas preparatorias para el acceso a la educación superior. En esta escuela comenzó su trabajo editorial en el periódico escolar del centro, publicado desde 1888 con el título de The jabberwock (El galimatazo, personaje proveniente de la fantasía de Lewis Carrol). Allí aprendió tres aspectos disciplinares que fueron de incalculable valor para ella: «La precisión, el sentido de prioridad, del orden en las tareas a realizar y la habilidad de organizar la propia programación que hace que las revistas lleguen a tiempo.» (Harris, 2004, p. 13).

A partir de 1904 continuó sus estudios en la School of Library Science del College Simmons School, institución dedicada exclusivamente a la formación femenina. El campo de la biblioteconomía era uno de los pocos desempeños profesionales especializados en los que las mujeres asumían un papel de liderazgo. Una de sus profesoras de entonces, posteriormente directora de la Simmons College Library School, June Richardson Donnely, la describió como «alta, delgada, una brillante joven de ojos oscuros, cuya inteligencia era una oriflama ansiosa a cualquier grupo del que formaba parte» (Donnely, 1936, p. 44).

El mismo año que finalizó sus estudios trabajó como editora y compiladora de 
la obra The New England Historical and Genealogical Register, incorporándose poco después al departamento de Fine Arts de la Boston Public Library. Esta experiencia previa permitió a Theodora ocupar la vacante como asistente en el departamento que dejó su hermano Fiske², estudiante de la Harvard Architecture School graduado en 1909, al recibir la codiciada Beca Sheldon, que tenía como finalidad complementar la formación de los estudiantes con una estancia en al extranjero. Theodora recibió entonces el encargo de la puesta en marcha de la biblioteca de la Harvard Landscape Architecture School.

Se trasladó a vivir a Cambridge, la pequeña ciudad frente a Boston que aspiraba a convertirse en el referente del conocimiento y la investigación en Estados Unidos como la ciudad homónima inglesa a la que debía su nombre. Para ello contaba con el tirón de sus dos universidades más prestigiosas: la veterana Universidad de Harvard, inspirada en el sistema de los colleges ingleses, y el Massachusetts Institute of Technology, enfocado a la formación técnica en ingeniería, construcción, mecánica y química. En Cambridge Theodora adquirió su propio apartamento y, por primera vez, disfrutó de la independencia en toda su dimensión. El hecho de vivir independiente y sola, aparentemente banal en el entorno masculino, se convertía en un auténtico logro en el contexto de su tiempo.

Theodora quedó "fascinada por el landscape architecture», utilizando la expresión reflejada en la correspondencia con su hermano Fiske 3 . Y así, en una primera declaración de intenciones, se propuso realizar un estudio sistemático de los parques metropolitanos norteamericanos. Esta actitud de curiosidad y entusiasmo, acompañada de una inusitada rigurosidad en el trabajo, fueron cualidades que mantuvo a lo largo de toda su carrera profesional.

Su responsabilidad profesional consistía en recoger todos los recursos disponibles sobre el landscape architecture, a los que pronto añadió los del city planning, y ponerlos a disposición de los profesionales en formación. El primer contacto con el city planning fue a través de su labor de clasificación de la voluminosa biblioteca personal de James Sturgis Pray, director del departamento de Landscape Architecture y titular de la cátedra Charles Eliot desde 1915. Esta cátedra había sido establecida en 1903 por la familia de Nelson Robinson, que también donaría el Robinson Hall y la Nelson Robinson Jr. Travelling Fellowship, en memoria de su hijo. James Sturgis Pray poseía buenos fondos relativos al city planning. Además, acababa de incluir en 1909 una asignatura dedicada al city planning en el programa de Landscape Architecture.

Desde la planta baja del Robinson Hall, Theodora Kimball comenzó la publicación de sus trabajos, editoriales, artículos, revisiones bibliográficas, reseñas y co-

2 Sobre Fiske Kimball véase: Fiske Kimball Papers 1874-1955, Archivo del Museo de Filadelfia. http:// www.philamuseum.org/pma_archives.

3 El epistolario de Theodora con su hermano, depositado en el Museo de Bellas Artes de Filadelfia, es una de las principales fuentes de información personal y profesional que utilizó Heidi Hohmann en su artículo "Theodora Kimball Hubbard and the "Intellectualization" of Landscape Architecture, 1911$1935 "$. 
laboraciones. El mismo edificio albergaba la sede de la Faculty of Architecture, que desde 1914 comprendía la Landscape Architecture School y la Architecture School.

En estos años iniciales, las colaboraciones de Theodora con Pray se centraban en el city planning y en el campo del landscape architecture con sus colaboraciones con el profesor auxiliar Henry V. Hubbard (1875-1947). Hubbard, discípulo y colaborador de Frederik Law Olmsted, Jr. (1870-1957), primer director de la Harvard Landscape Architecture School, se había incorporado a la docencia como lecturer en 1906 y a partir de 1921 fue professor del departamento de Landscape Architecture. Objetivamente, Theodora tenía una posición laboral nada convencional en la universidad dado que no pertenecía al cuerpo docente, pero tampoco era estrictamente una bibliotecaria. La singularidad no venía dada por su responsabilidad, puesto que también la biblioteca de la Harvard Architecture School estaba bajo dirección femenina, Elizabeth D. Clarke, a la que sucedió Ruth V. Cook en 1919, sino por su actitud y grado de implicación. No se centró sólo en la responsabilidad de la incorporación y la clasificación del material bibliográfico acopiado. A pesar de carecer de una formación técnica y profesional específica, su manejo de las fuentes de información suplía esa carencia y era capaz de estimular con su trabajo a todos, incluidos aquellos que sí disponían de ella. Se implicaba muy directamente en los estudios de los alumnos, manifestando una destacable empatía con ellos, por lo que pronto se encontró rodeada de profesores y alumnos universitarios que vieron con interés su labor y con los cuales logró crear un estimulante ambiente de trabajo.

En 1917, Theodora Kimball recibió un Master of Science por el Simmons College, justificado por su creativo trabajo en la clasificación de materiales relativos al city planning. Además, preparó una tesis sobre paisajismo inglés y una selección de listados sobre city planning para una lectura más popular destinados a las bibliotecas públicas (Donnely, 1936, p. 44).

Unos años más tarde, en 1924, tuvieron lugar varios acontecimientos trascendentales en la vida de Theodora Kimball. Después de una intensa relación profesional y personal, contrajo matrimonio con Vincent H. Hubbard, se produjo el fallecimiento de su padre y renunció a su puesto de bibliotecaria por motivos de salud, pasando a ser sustituida por su colaboradora y protegida desde 1918, Katherine MacNamara ${ }^{4}$, la cual se mantuvo en su cargo durante décadas. A partir de ese momento, continuó colaborando con la biblioteca en calidad de asesora y firmó sus publicaciones como bibliotecaria honoraria del American Institute of Planning. En sus trece años al frente de la biblioteca de la Harvard Landscape Architecture School, dejó una tradición que fue emulada por las mujeres que asumieron cargos de perfil similar, como Lucille Keck, bibliotecaria en Chicago de la American Society of Planning Officials, y Mary Vance, responsable del Council of Planning Librarians, que expandieron su potencial investigador editando miles de bibliografías.

A partir de 1930 la actividad de Theodora declinó bastante debido al deterioro de

4 Katherine MacNamara pasó a ser responsable de la biblioteca de la Harvard Design School tras el retiro de Ruth V. Cook en 1956. 
su estado de salud, aunque seguía figurando como colaboradora en las numerosas iniciativas con las que estaba comprometida. Pasó sus últimos años muy delicada de salud en la casa familiar de Spafford Road, en Milton, Massachusetts, diseñada por su hermano Fiske, hasta su fallecimiento a finales de 1935. El artículo in memorian publicado por la revista Landscape Architecture ofrece un testimonio muy preciso de su talante: "Theodora Kimball Hubbard fue una profesional de gran distinción. Postrada en cama durante algunos años por enfermedad, [...], recibiendo el reconocimiento de personalidades de muchos países [...]. Tanto en su vida profesional como en su largo círculo de amistades, Mrs. Hubbard era una amiga infalible. En sus subordinados ella inspiró una lealtad no distinta a la que les dio ella.» (Landscape Architecture, 1936, p. 55).

Theodora Kimball participó directamente en las iniciativas que dotaron de identidad propia al urbanismo norteamericano, consolidado tras un complejo proceso de formación. Desde la Harvard Landscape Architecture School Library estudió a los pioneros del urbanismo, colaboró en el paso del landscape architecture al regional planning y con sus sistematizaciones de la abundante información bibliográfica generada en su entorno, permitió la difusión del zoning y la internacionalización de la singular experiencia norteamericana tras la Primera Guerra Mundial.

\section{Los pioneros del landscape architecture: Andrew Jackson Downing, Frederik Law Olmsted y H. S. W. Cleveland}

El malogrado Andrew Jackson Downing (1815-1852), Frederik Law Olmsted (1822-1903), y Horace William Shaler Cleveland (1814-2900) eran considerados la terna por excelencia de los paisajistas norteamericanos, por lo que no es de extrañar que cualquier aproximación al ámbito del paisajismo los tuviera en cuenta en mayor o menor medida. De Cleveland destacaba su plan para Mineápolis, un proyecto de ordenación urbana global realizado en 1883 y basado en un sistema integrado de áreas verdes sobre la previsión de la construcción de 32 kilómetros de parkways, tal y como se recogía en «H.W. S. Cleveland. An American pioneer in Landscape Architecture and City Panning» (Kimball, 1930, pp.92-111), artículo con el que se pretendió el reconocimiento a un paisajista cuya labor se situó en el momento de la transición del paisajismo romántico al planeamiento profesional y cuya figura fue inexorablemente eclipsada por Frederik Law Olmsted, Sr.

En 1920 Frederick Law Olmsted, Jr. se propuso publicar los escritos de la carrera profesional de su padre con motivo del centenario del nacimiento y ofreció a Theodora Kimball realizar los trabajos de edición. Theodora era ya entonces una figura reconocida por sus trabajos bibliográficos y estaba acostumbrada al tratamiento de las fuentes de información, controlando todos los manuscritos depositados en su biblioteca.

La publicación se dividió en dos volúmenes. El primero, correspondía a la edi- 
Tomo XVI.

Habana 30 de Abril de 1921

Núm. 9

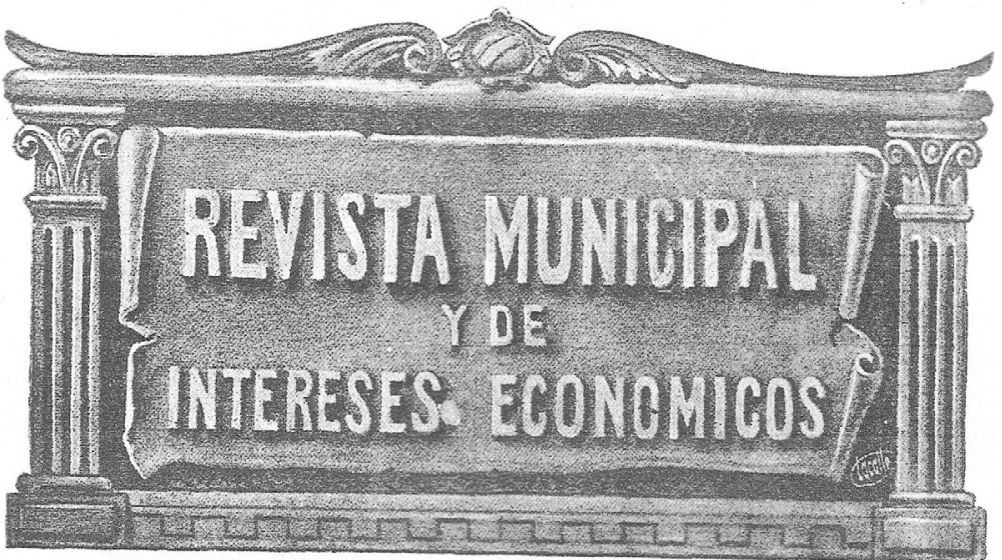

Director Propietario: Dr. F. Carrera Justiz

Profesor de Gobjerno Municipal e Historia de las Instituciones Locales de Cuba en la Escuela de Derecho-Público de la Universidad de la Habana.

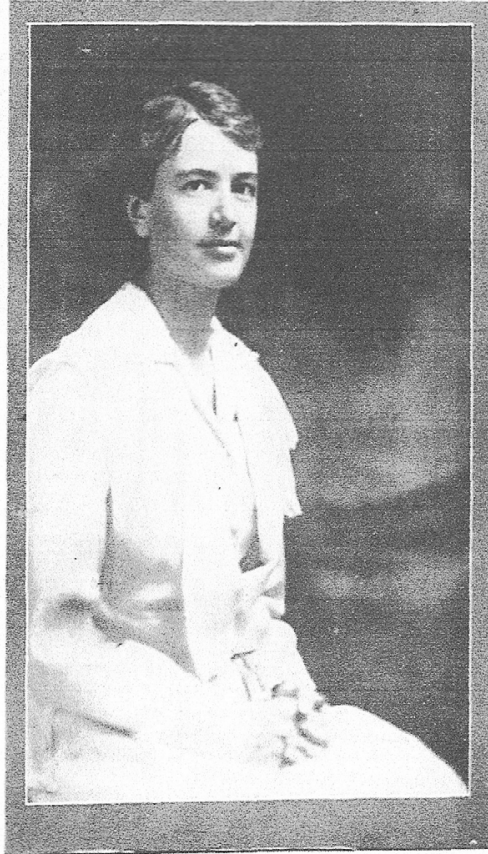

THEODORA KMEALL

Eibliotecaria de la Univers

Figura 1. El Urbanismo en los Estados Unidos. Theodora Kimball.

Fuente: Carrera Justiz, F. Portada. Revista Municipal y de Intereses Económicos, XVI (9) abril, 1921 
ción de los papeles profesionales de cuarenta años como landscape architect de Frederick Law Olmsted Sr., y fue publicado en 1922 con el título Frederick Law Olmsted. Landscape Architect 1822-1903. No era una biografía al uso, sino la historia del personaje construida sobre la reproducción parcial de sus textos profesionales, en su mayoría epistolares, de los años de desarrollo de su carrera profesional. El minucioso relato, que incluía una extensa y exhaustiva cronología donde se sucedían mes a mes los hechos acaecidos a lo largo de su vida, constituye una muestra del ingente trabajo realizado. En él no se planteaba una visión crítica sobre las aportaciones de Olmsted, sino un trabajo de carácter más bien expositivo, donde las relaciones con A. J. Downing merecieron un capítulo.

La influencia que tuvo como referente Central Park en los proyectos de parques urbanos de las ciudades norteamericanas justificaba para los editores la separación del trabajo en un segundo volumen, cuya publicación se demoró hasta 1928. A. J. Downing, auténtico ideólogo y promotor del parque neoyorkino, amigo y estímulo de Olmsted Sr., fue quien le suscitó el interés por el parque de Nueva York y le introdujo a Calvert Vaux, coautor de la propuesta Greensward, ganadora del concurso para la ejecución del Central Park de 1857, y ello a pesar de sus diferencias ante el landscape architecture. Sobre este vínculo profesional Theodora Kimball escribió: «llama poderosamente la atención observar el contraste entre el método de discutir problemas de paisaje, apriorístico y doctrinario de Downing, y el método habitual del Sr. Olmsted, que era prever francamente la peculiaridad de hechos de cada situación como un problema individual que había que resolver por sus propios méritos de manera individual, y después de probar, y tal vez corregir, sus conclusiones razonando, volver a encontrar principios de coherencia tanto con los hechos y las intuiciones artísticas presente en el caso en particular, con otros principios y teorías aceptadas por él como verdaderas.» (Olmsted \& Kimball, 1922, p. 2.).

El monográfico Central Park as a work of art and as a great municipal enterprise, 1853-1895, contó para su publicación con el soporte económico de la Russell Sage Foundation, la cual concedió una beca especial a los editores de los papeles de Olmsted para producir «un monográfico sobre Central Park no sólo dedicado al diseño, sino a la compleja historia de su concepción, su diseño, su construcción, como un gran espacio natural completamente artificial, y el desarrollo de su gestión hasta los años ochenta [del siglo XIX]. El volumen no será entonces sólo dedicado a la contribución de Olmsted como diseñador, sino que pretende mostrar el esfuerzo colaborativo para una comunidad democrática» (Olmsted \& Kimball, 1928, p. v). La definición del parque como municipal enterprise era una muestra del intento de volcar en la ciudad el espíritu democrático norteamericano como principal exponente de la política de gestión de la responsabilidad municipal, dada la magnitud del impacto económico generado por este tipo de intervenciones en las urbes.

Este proyecto editorial se enmarcaba en los esfuerzos de inversión económica que estaba realizando en la ciudad de Nueva York la Russell Sage Foundation ${ }^{5}$, en

5 Trabajo de investigación en curso sobre Olivia Sage y la Russell Sage Foundation. 
cuyos estatutos se establecía que la ciudad debía ser la beneficiaria de, por lo menos, una cuarta parte de su presupuesto, y que daría lugar al desarrollo del proyecto Regional Plan of New York and its Environs. Para este plan se planteó una primera fase de civic survey sobre el estado económico, industrial, físico, legal y social y de condiciones de vida en la ciudad a escala regional. Frederick Law Olmsted, Jr., John Nolen, Harlam Bartolomew, George B. Ford y Edward H. Bennet fueron los profesionales que colaboraron con Thomas Adams (1871-1940)6, responsable del trabajo durante siete años y al que Theodora agradeció su colaboración en su prefacio del libro.

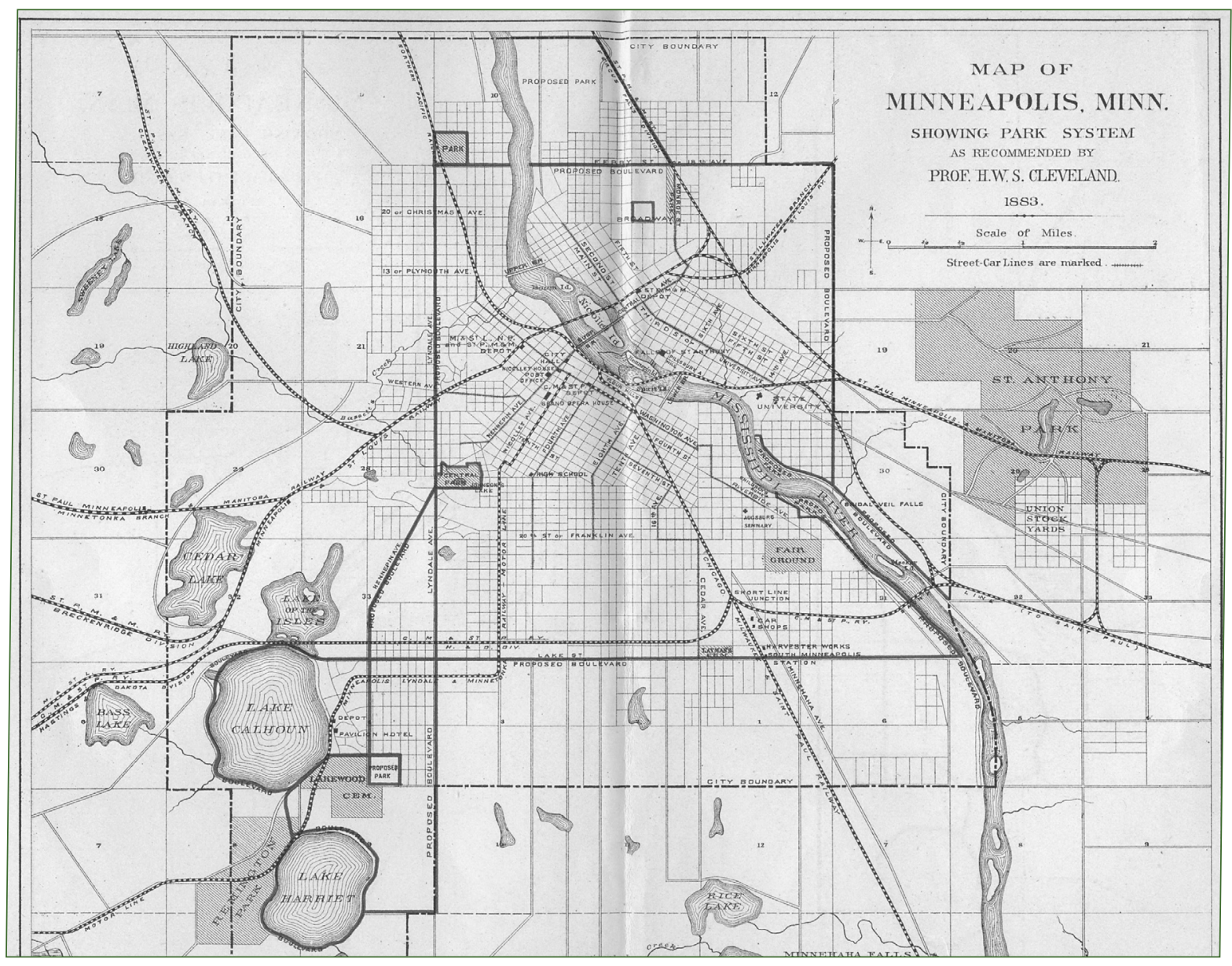

Figura 2. Mapa de Mineápolis mostrando su park-system tal y como recomendaba el prof. H. W. S. Cleveland, 1883.

Fuente: Kimball, T. H.W. S. Cleveland. An American pioneer in Landscape Architecture and City Planning. Landscape Architecture, (20) enero, 1930, p. 105.

6 Se publicó la extensa guía, en doce volúmenes, para el desarrollo de la región metropolitana de Nueva York, entre los que se incluía el trabajo de Clarence Perry sobre las unidades vecinales. 


\section{Acerca del landscape architecture en la Harvard Landscape Architec- ture School}

Los estudios de landscape architecture se habían iniciado en Estados Unidos en la Universidad de Harvard en el año 1900 bajo la dirección de Frederik Law Olmsted, Jr., en la que se mantuvo hasta 1908. El órgano de difusión de la American Society of Landscape Architects (1899) era la revista Landscape Architecture, creada en 1910 por Henry. V. Hubbad, Charles Downing Lay, primeros graduados de la Harvard Landscape Architecture School, y Robert Wheelwright, compañeros todos durante un tiempo en un despacho profesional de landscape arquitecture en Nueva York.

Claro testimonio del estado iniciático de su profesionalización en la primera década del siglo XX fue el artículo publicado en la revista Landscape Architecture «The arrangement of the professional collections of a Lanscape Architect" (Hubbard y Kimball, 1913). Era un texto de carácter eminentemente práctico que versaba sobre el archivo profesional del variado material de consulta que implicaba la labor del landscape architect y que incluía cartografías, fotografías, postales, cartas, diapositivas, etc., estableciendo además una propuesta de clasificación de los materiales basada en conceptos todavía no reglados ${ }^{7}$.

El artículo se completaba con una bibliografía de referencia considerada necesaria para una oficina profesional en la que el city planning contaba con una demarcación específica "para aquellos interesados». Se incluía el districting o zoning en el apartado correspondiente a la composición urbana. (Hubbard y Kimball, 1913, p. 78). Con este artículo se iniciaba la intensa colaboración de Theodora Kimball con la revista, que incluía sistemáticas actualizaciones de la sección bibliográfica, la publicación de algún artículo de fondo y reseñas de libros. John Nolen apuntó años más tarde: «El reportaje anual de información de ciudades realizado por Theodora Kimball Hubbard es uno de los más valiosas contribuciones del landscape architecture al progreso del urbanismo en los Estados Unidos durante las últimas dos décadas.» (Nolen, 1930, p. 296).

La Harvard Landscape Architecture School tuvo en el libro An Introduction to the Study of Landscape Design (Hubbard \& Kimball, 1917) su manual de referencia, que fue dedicado por sus autores a los fellow students. Partía de la definición de landscape architecture como «el arte de la adecuación de la tierra para el uso y el disfrute humano. El arquitecto paisajista diseña y asesora en relación con la intervención, y dirige el desarrollo de la tierra y los objetos sobre ella en relación con espacios abiertos privados y jardines, instituciones, parques públicos, parques infantiles y plazas, cementerios, calles y parkways, comunidades residenciales, y los problemas de planificación urbana y regional» (Hubbard \& Kimball, 1917, p. 1). El landscape architecture era tratado en toda la amplitud que requería como nuevo campo profesional, desde los elementos que lo componen, las escalas implicadas - desde el jar-

7 Ver H. Hohmann. Theodora Kimball Hubbard and the "Intellectualization" of Landscape Arc hitecture, 1911-1935. Landscape Journal, 25 (2) septiembre, 2006, pp. 169-186. Doi: 10.3368/1j.25.2.169 
dín de la vivienda a los grandes parques naturales - y la gestión del suelo, llegando a considerar no sólo el mundo de los sentidos, sino también el de las emociones. Se abordaba también el fundamento filosófico y estético que soportaba la obra desde las bellas artes, como puede leerse, según el resumen de sus autores, en frases como la que sigue: «la belleza es el placer considerado como la cualidad de una cosa». Se añadía además el valor ético y propositivo que representan los parques, dado que su mera existencia era capaz de promover el comportamiento moral en la comunidad y éste era uno de los principales argumentos que justificaban su existencia. Sin embargo, esta visión del paisaje adolecía de una referencia al contexto social desde otros parámetros.

La obra no era considerada por sus autores como un compendio de utillaje para su aplicación práctica: «no es un libro de reglas ni tampoco persigue establecer una teoría estética» (Hubbard \& Kimball, 1917, p. vii), sino un completo repertorio de ilustraciones, muchas de las cuales eran del propio Hubbard, con las cuales el profesional podía ver cómo los problemas a los que se tenía que enfrentar se habían solventado con anterioridad. Los ejemplos clásicos alemanes, italianos, franceses y españoles, con la Alhambra, el jardín japonés o las creaciones norteamericanas, son algunos ejemplos que recoge el estudio, destacando la influencia alemana que estaba presente a través de la admiración de Charles Eliot por el parque Muskau de Pückler. Para los Hubbard no existía el concepto de tabula rasa en el conocimiento.

La publicación titulada Landscape architecture; a comprehensive classification scheme for books, plans, photographs, notes and other collected material, with combined alphabetic topic index and list of subject headings (Hubbard \& Kimball, 1920), considerada por sus autores como complementaria del City planning classification (Pray \& Kimball, 1913), pretendía reafirmar la intrínseca relación entre el landscape architecture y el city planning, que «deberían utilizarse conjuntamente, como en la Harvard Landscape Architecture School Library, donde ambos conceptos son desarrollados y aplicados simultáneamente. [...] Numerosas referencias cruzadas con la numeraciones correspondientes al city planning han sido hechas, con la indicación de que se ha probado el lugar conveniente para clasificar el material cubierto por ambas clasificaciones.» (Hubbard \& Kimball, 1920, p. 7).

\section{Acerca del city planning en la Harvard Landscape Architecture School}

En 1912 se publicó el primer gran listado recopilatorio de todas las publicaciones relativas al city planning a cargo del Gobierno norteamericano. En Special Libraries, boletín de difusión de la Special Libraries Association, se publicó una extensa recopilación de 72 páginas, denominada Check List on City Planning, bajo la dirección editorial del H. B. H. Meyer, bibliotecario jefe de la división de Bibliografía de la Biblioteca del Congreso de los Estados Unidos de América, basada en los trabajos de la Harvard Landscape Architecture School -la instauración oficial de la Harvard 
Landscape Architecture School Library no se produría hasta 1913-. La propuesta de clasificación fue establecida en base a dos grandes apartados: el primero, con el contenido general de materias, y el segundo, sobre la literatura producida acerca de las ciudades, incluyendo entremezcladas referencias norteamericanas y extranjeras, especialmente de Francia, Inglaterra y Alemania. También se incluían aportaciones puntuales de otros países, como la obra española Teoría General de la Urbanización y aplicación de sus principios y doctrinas a la reforma y ensanche de Barcelona (1867) de Ildefonso Cerdá.

Check List on City Planning se presentó como un avance del esquema de clasificación del city planning, sobre el que se estaba trabajando en el departamento de Landscape Architecture de la Universidad de Harvard, que daría como resultado A City-Planning Classification: a comprehensive analysis of the subject arranged for the classification of books, plans, photographs, notes and other collected material, with alphabetic subject index (Pray y Kimball, 1913), publicado por Harvard University Press, editorial universitaria fundada ese mismo año. La publicación consistía en una extensa y minuciosa clasificación, de base numérica, de conceptos y variables que afectaban al city planning, en la que el zoning tenía asignado un epígrafe propio. La entidad que aglutinaba a los interesados en el city planning era el American City Planning Institute (1917). Contaba con Frederick Law Olmsted, Jr. como primer presidente, y en 1919 se incorporó Theodora Kimball como bibliotecaria honoraria, coincidiendo con su ingresó en la American Society of Landscape Architects. Las National Conference on City Planning, iniciadas en 1909, se convirtieron en importantes foros de reflexión del urbanismo norteamericano.

El city planning se desarrolló a nivel universitario en el seno de los estudios de landscape arquitecture. El contenido de los cursos pioneros de Sturgis Pray en la Harvard Landscape Architecture School se presentó en la publicación A City-Planning Classification: Preliminary Outline, avance de un compendio final más ambicioso que fue distribuido entre especialistas con el fin de que realizaran los comentarios oportunos. Frederick Law Olmsted, Jr. fue consultado acerca del survey; Flavel Shurtleff sobre legislación; Charles W. Killam, de la Harvard School of Architecture, sobre tipos de edificios. A ellos se añadían los nombres de John Nolen, James Ford, profesor del departamento de Social Ethics y Josef Brix y Felix Genzmer, directores del seminario anual sobre problemas urbanísticos y construcción cívica de la Technische Hochschule de Berlín-Charlotemburgo, entre otros (Pray y Kimball, 1913).

Este folleto constituía un buen exponente de una manera de abordar el urbanismo genuinamente americana. El city planning era utilizado en su sentido más amplio y quedaba definido «como el control inteligente y guía de la conformación física, crecimiento y alteración de ciudades, o partes de entidad, considerados en su totalidad. Incluyendo el planeamiento de ciudades, suburbios, pueblos y cualquier distrito considerable urbano, suburbano o incluso rural». Se partía de la consideración de la ciudad, incluidos sus aspectos territoriales, entendida como un centro alrededor del cual se estructuraba el vasto territorio americano. Se la consideraba 
una entidad global en la que cada aspecto analizado adquiría su propio carácter sólo en relación con el todo. Esas relaciones no eran sólo físicas, sino que abarcaban también las dimensiones sociales, económicas y de administración y gestión de los recursos.

Desde 1923 la Harvard Landscape Architecture School ofrecía un máster en city planning cuyos cursos técnicos fueron liderados por Pray y Hubbard. A ellos se sumaban las conferencias de profesionales de prestigio, actividad que por otra parte ya venía realizándose desde hacía tiempo en el Robinson Hall, como lo pone de manifiesto la conferencia "City planning in India», dictada por Patrick Geddes unos meses antes del inicio de curso. La idea principal era convertir estos estudios en el equivalente a los desarrollados en el departamento de Civic Design de la Universidad de Liverpool, en el University College de Londres (1914), en el departamento de diseño urbano de la Ecole des Hautes Etudes Urbaines (1913) del Institut d'Histoire, de Géographie et d'Économie Urbaines de París, en el seminario de la Technische Hochschule de Berlín-Charlotemburgo (1908), y en las iniciativas que tenían lugar en Bélgica.

Poco a poco los estudios de landscape architecture y city planning fueron delimitando sus ámbitos de responsabilidad hasta que su vinculación docente se rompió en 1929, cuando en el seno de la Universidad de Harvard se creó la Graduate School of City Planning bajo la dirección de Hubbard, hasta entonces profesor de la asignatura de Landscape Construction. La experiencia académica del city planning como rama de estudio independiente contaba con un precedente de corto recorrido en el mundo universitario norteamericano: la cátedra de urbanismo de la Universidad de Illinois en Chicago, creada en 1913 para Charles Mulford Robinson como professor de Civic Design, hasta que tras su fallecimiento en 1917 fue reincorporada al departamento de Landscape Gardering.

El activo municipalismo se aglutinaba en torno a la National Municipal League, que publicaba la revista Municipal Review. Con ella colaboraba Theodora Kimball con su «Brief review of city planning in the United Status» (Kimbal, 1921, pp. 3950), unos listados recopilatorios actualizados anualmente desde 1917 a 1926, cuyos apartados son un exponente de su concepción del city planning: las diferentes escalas regional, estatal y nacional, el planeamiento preventivo y correctivo, la necesidad de una ley integral, el survey, el plan, la financiación, el programa constructivo, la educación del público para su puesta en valor, las comisiones oficiales y de asesoramiento, el desarrollo de los proyectos, el zoning, el control de las subdivisiones de manzanas, el housing, la industria, los transportes y las terminales, los campos de juegos y los parques, y los reports integrales a los que se añadía un apartado de estudios más específicos. En una de sus definiciones de city planning escribió: «Cuando hablamos de planificar la ciudad, hablamos del acondicionamiento para la conveniencia y confort de los más grandes o más pequeños grupos de hogares, tiendas carreteras y parques de tal modo que la gente que vive en las ciudades tenga el mejor acceso posible a la salud, la riqueza y la felicidad» (Kimball, 1923, p. 3). 


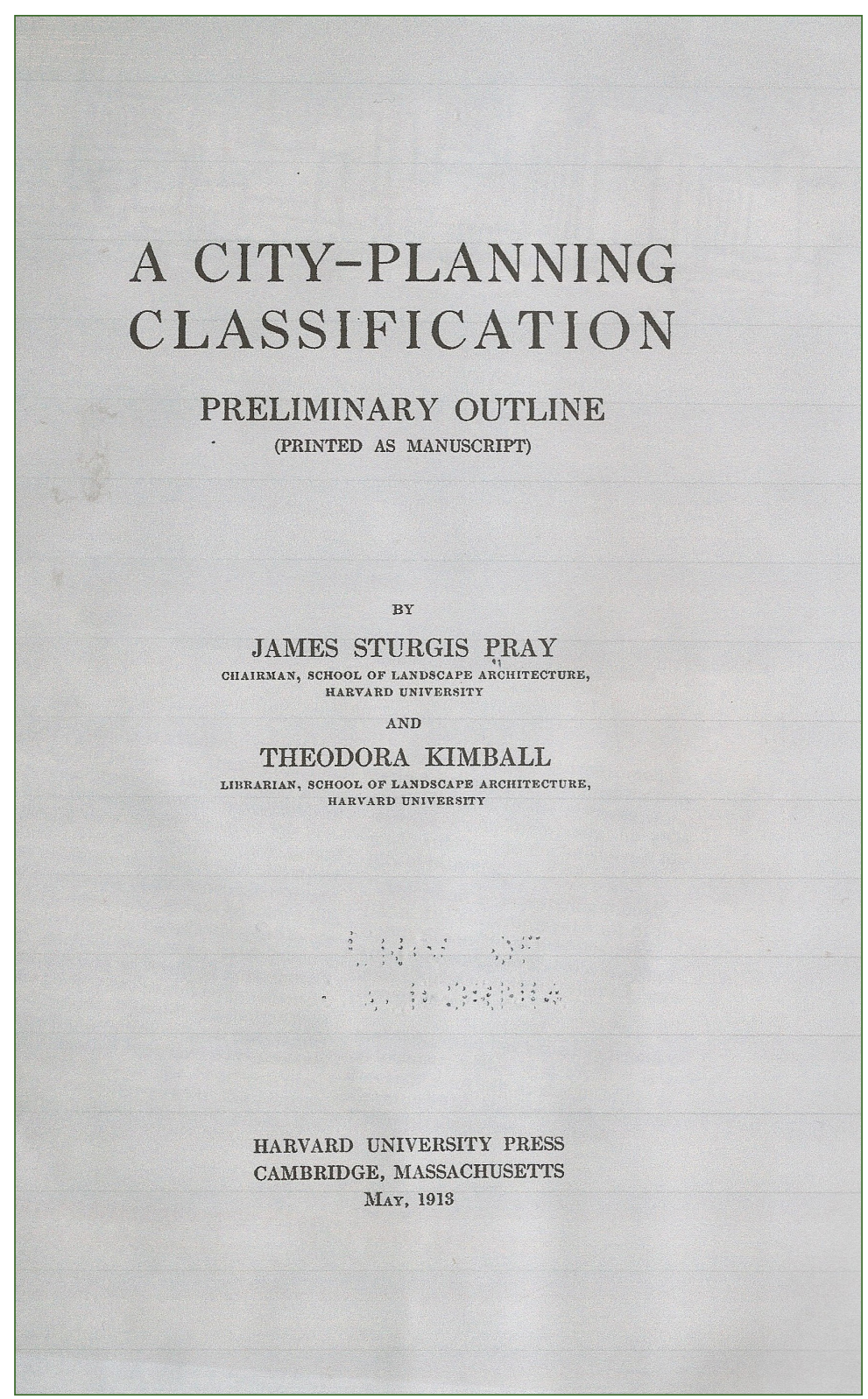

Figura 3. Portada de la publicación A city planning classification, preliminary outline.

Fuente: Sturgis, J. \& Kimball, T. A city planning classification, preliminary outline. Cambridge: Harvard University Press, 1913.

\section{El urbanismo como acto de comunicación: los reports}

Por report se entendía cualquier tipo de publicación que abordara una amplia temática referida a la ciudad desde distintos aspectos, como las limitaciones de alturas en los edificios, el sistema de parques, las infraestructuras de transportes o las perspectivas de futuro. Los promotores de estas iniciativas eran asociaciones pri- 
vadas de ámbito municipal, juntas de carácter consultivo como los Town Planning Committees y muchas veces los propios alcaldes. El caso paradigmático del report en formato de libro fue el Plan of Chicago de 1909, patrocinado por el Commercial Club of Chicago. Con una tirada de 1.650 ejemplares, tenía como finalidad la difusión del plan de Chicago, primer plan americano financiado por una entidad privada, el Commercial Club.

"A brief survey of recent city planning reports in the United States» (Kimball, 1912) es el título de la relación de reports de veintiocho ciudades norteamericanas publicados en Estados Unidos entre 1910 y 1912. Fueron elaborados por técnicos entre cuyos nombres destacaban John Nolen, Charles Mulford Robinson y Bion J. Arnold, si se trataba de temas relativos a las infraestructuras de transportes y luego convenientemente adaptados a su finalidad. Así, el informe de John Nolen elaborado para la pequeña ciudad de Schenectady, Nueva York, fue transformado en el panfleto «A Penny a Week for Parks and Playgrounds» como campaña publicitaria por parte de su alcalde.

En el artículo «Personality and Human Beings in Town-Planning Work» (Kimball, 1920, p. 609), escrito para la revista Amercican City, Theodora Kimball expresaba su concepción del urbanismo como un acto de comunicación, en el que interactuaban dos agentes, la sociedad y el profesional, cuya principal labor era saber interpretar lo que demandaba la sociedad y ser capaz de transmitir en un lenguaje hablado o escrito, la idea que respondiera a esa demanda. Frente al debate sobre la técnica en la Scientific City, liderado por George B. Ford, y el arte cívico como exponente arquitectónico de la City Beautiful, que representaba el plan de Chicago de Daniel H. Burnham y Edward H. Bennett, el urbanismo se trasladaba al plano de la interacción social. La sociedad dejaba de ser considerada como agente pasivo y mero receptor de las acciones propuestas a ser un agente activo capaz de promover iniciativas y un valedor necesario de las propuestas generadas por los profesionales.

El objetivo era saber vender ?tomando prestado el lenguaje de los negocios? el plan de urbanismo, reconociendo que «la atención es necesaria para comprender, comprender para interesar e interesar para apoyar las ideas que se pretendan poner en marcha» (Kimball, 1922, p. 198). Se deduce así la importancia de las campañas formativas entre los ciudadanos. Y el mejor medio con el que contaba el urbanista era el report, el documento a modo de panfleto, o incluso libro, con el que divulgar la información relativa a las propuestas sobre el desarrollo urbano de la ciudad. De su modo de presentación, de su tipo de lenguaje, de la accesibilidad de sus contenidos y de la adecuación al público al que iba destinado, dependía en gran medida la deriva que tomasen las propuestas. El urbanista era entendido en su dimensión comunicadora y, por tanto, con sus limitaciones en sus actitudes y aptitudes, pero dotado de un necesario potencial creador. "Lo que necesitamos es más sangre roja» (Kimball, 1922, p. 609), apuntó Theodora Kimball recogiendo la expresión utilizada en una de las sesiones de la National Conference on City Planning. Para entender la importancia de estos reports, se debe tener en cuenta el contexto de carácter propo- 
sitivo intrínseco al planeamiento norteamericano hasta la irrupción del zoning, que era de carácter imperativo.

En Municipal accomplishment in city planning and published city plan reports in the United States (Kimball, 1920), uno de los trabajos de mayor repercusión de Theodora Kimball, se actualizaban, perfectamente estructuradas, todas y cada una de las iniciativas de carácter urbanístico publicadas por cada uno de los municipios del país. La base metodológica del trabajo partía de la encuesta, el survey, en la que se solicitaban los datos relativos a las acciones realizadas por el municipio para ser incluidos en la publicación. La encuesta era enviada a cada responsable municipal y el municipio era libre de contestar o no. Este trabajo fue auspiciado por la National Conference on City Planning de 1920, que se propuso disponer de una foto fija de la práctica del city planning a lo largo y ancho de los Estados Unidos de América o más precisamente de sus publicaciones. La finalidad no era otra que hacer partícipe a los municipios americanos de la necesidad de actuar en sus respectivos ámbitos urbanos.

\section{La internacionalización del city planning tras la Primera Guerra Mundial}

En el trabajo bibliográfico elaborado por Theodora Kimball para la National Conference on City Planning de Boston (1915), donde la presencia internacional fue manifiesta, su autora especificaba que «los primeros reconocimientos de la literatura del city planing eran los reports de Frederik Law Olmsted, Sr., y el Landscape Architecture as Applied to the Wants of the West de H. W. S. Cleveland; a los que se añadían los trabajos realizados en Les Promenades de Paris de Charles-Adolphe Alphand en Francia, Stadterweiterungen in technischer, baupolizeilicher und Wirtschaftlicher Beziehung de Reinhar Baumeister en Alemania y, más tardíamente, Garden Cities of To-morrow de Ebenezer Howard.» (Kimball, 1915, p. 3). La recopilación bibliográfica recogía las publicaciones que circulaban por Europa ${ }^{8}$, especialmente Alemania, Inglaterra y Francia. En cuanto a las revistas, constataba que las dedicadas íntegramente al urbanismo en ese momento eran sólo cinco: la americana City plan (1915), las inglesas Garden cities and town planning (1906) y Town planning review (1910) y las alemanas Gartenstadt (1907) y Der Statebau (1904).

La Primera Guerra Mundial supuso para el urbanismo la apertura de un nuevo frente de trabajo, como era la difusión de las iniciativas de reconstrucción que se estaban llevando a cabo en los países aliados, principalmente Inglaterra, Francia

8 Las referencias españolas citadas fueron un panfleto sobre la reforma interior de Barcelona (1907), la revista Civitas (1920), editada por la Sociedad Cívica de la Ciudad-jardín de Barcelona, y el artículo Madrid's scheme of city planning. American Architect, 118 agosto, 1920, p.173. Añadía además una referencia específica a la Ciudad Lineal de Arturo Soria. 
y Bélgica ${ }^{9}$ y, por tanto, la intensificación de los contactos internacionales. Organismos oficiales como el U.S. Bureau of Industrial Housing and Transportation acopiaban informaciones relativas a estas experiencias. Theodora Kimball fue nombrada bibliotecaria para las referencias bibliográficas. Fue la habilidad demostrada en la organización de este trabajo y su espíritu enérgico las cualidades que llamaron la atención del entonces secretario del Departamento de Commerce del Gobierno norteamericano, Herbert Hoover.

Un personaje clave en el conocimiento de las acciones llevadas a cabo en la reconstrucción europea fue George Burdett Ford, que se encontraba en Francia con motivo de sus trabajos para la reconstrucción de las ciudades europeas en la Renaissance des Cités ? ?una asociación que estableció una oficina de cooperación, de información y documentación de los trabajos de reconstrucción económica, social y arquitectónica de las ciudades francesas.

En la Conferencia Interaliada de París de 1919 se presentó el libro City Planning Progress in the United Estates (1917), obra dirigida por George B. Ford para el American Institute of Architects Committee on Town Planning, que recogía la descripción de la situación urbanística de 233 ciudades norteamericanas de más de 25.000 habitantes y algunos ejemplos significativos con una población inferior a esa cifra, que contó con la colaboración de Theodora Kimball, que figura en las actas de las conferencias interaliadas de París, y un año después en Londres, de la mano de Henry R, Aldridge, Alfred Pierre Agache y Ford. Esta obra fue el precedente inmediato de Municipal accomplishment in city planning and published city plan reports in the United States (Kimball, 1920). City Planning Progress fue difundida en España por el urbanista César Cort (Cort, 1919).

En ese camino de ida y retorno de esfuerzos de difusión del urbanismo, la Renaissance des Cites expuso parte de sus trabajos en el Robinson Hall de Harvard bajo los auspicios de la Harvard Landscape Architecture School en 1921, tal y como recogió el Harvard Crimson ${ }^{10}$.

Las publicaciones de Theodora constituían un claro referente del enfoque norteamericano de los temas municipales en los foros de encuentro europeos. Así, George Burdett Ford incluyó como referencia bibliográfica principal de su obra L'Urbanisme in practique, sus trabajos para la National Conference on City Planning de Boston de 1915. Y Patrick Geddes incluyó su trabajo de recopilación bibliográfico desarrollado en la revista City Planning entre la referencias norteamericanas presentes en su obra Cities in Evolution, junto con las aportaciones de Charles Mulford Robinson y John Nolen (Geddes, 1915, p. 406).

9 Theodora Kimball escribió tres artículos sobre la reconstrucción europea: The reconstruction of French and Belgican towns destroyed in the war. Landscape Architecture, 6 (4), 1918, pp. 165-171; Cityplanning problems in the reconstruction of French towns. Landscape Architecture, (8) enero, 1918, pp. 53-68 y Our British allies and reconstruction; a review of some recent writings, Landscape Architecture, julio, 1918, pp. 169-174.

10 The Harvard Crimson. 18 de junio de 1921. Show French exhibit all week. 


\section{Zoning Will Prevent This}

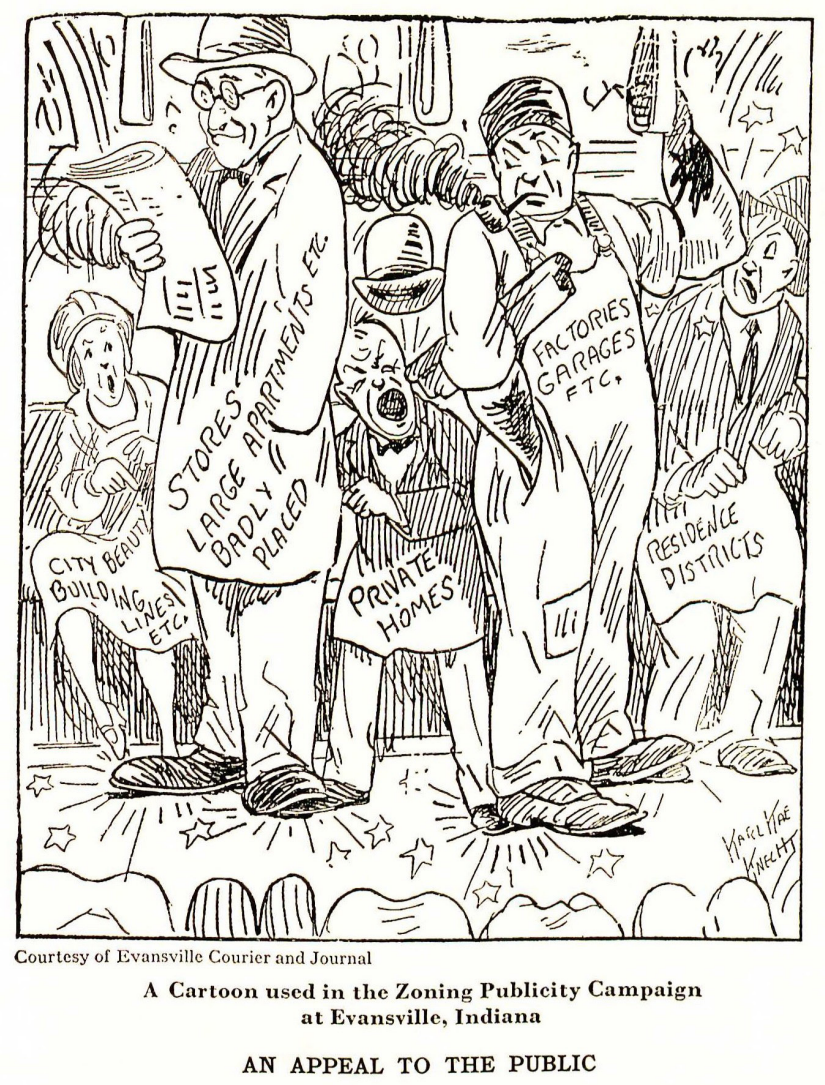

Figura 4. Una apelación al público. Una caricatura utilizada en la campaña publicitaria sobre el zoning en Evansville, Indiana.

Fuente: Kimball, T. y Hubbard, H. Our cities, to-day and to-morrow; a survey of planning and zoning progress in the United States. Cambridge, Mass.: Harvard University Press, 1929, p. 87.

Los ecos del trabajo de Theodora Kimball también llegaron a España, aunque con unos años de retraso. En la sección bibliográfica dedicada al urbanismo de la revista Arquitectura, a cargo del arquitecto Fernando García Mercadal, éste citaba en 1928 dentro del contexto de una visión general de los esfuerzos de la consolidación bibliográfica del urbanismo, cómo «la primera será debida a la colaboración del departamento de Landscape Architecture de la Universidad de Harvard con la Biblioteca Nacional ${ }^{11}$. El trabajo ha sido comenzado por miss Theodora Kimball, bibliotecaria del citado departamento de Harvard, a la cual ha sustituido después miss Catalina Mac Namara.» (Mercadal, 1928, p. 202). Dos razones aducía Mercadal para justificar las dificultades que convertían en una tarea siempre ardua la redac-

11 Se refiere a la Biblioteca del Congreso de los Estados Unidos de América. 
ción de una bibliografía completa de urbanismo: en primer lugar la abundancia de materiales y, en segundo lugar, la insuficiente delimitación del asunto. Superar estos condicionantes en base de un meticuloso trabajo fue lo que pretendió Theodora Kimball.

\section{Zoning has taken the country by storm (Kimball, 1922, p. 203)}

Oficialmente, durante los años veinte, el zoning - una vez desterrado el vocablo districting usado en los inicios-, trataba de organizar la ciudad mediante una regulación que la distribuyese en zonas diferenciadas, relacionando la altura de las edificaciones, los usos del suelo y las densidades de población.

Los Estados Unidos de América se rindieron ante el zoning, que no dejaba de ser una imposición, en lo que constituía una pequeña renuncia al modo de vida democrático a favor del bien común, haciéndolo prevalecer sobre los intereses privados. En 1929, tres quintas partes de la población urbana norteamericana vivían en una de las 754 comunidades dotadas de regulación de zoning en el uso del suelo, frente a las 100 de seis años antes; habría que añadir que 650 municipios contaban con comisiones de city planning (Hubbard, 1929, p. 3). La relación entre city planning y zoning no estaba tan claramente definida como a priori podría parecer. A diferencia del planning, que tenía un carácter consultivo, el zoning estaba dotado de un carácter impositivo, basado en el "poder de policía"12 asignado al Estado.

En 1922 Theodora Kimball fue requerida para formar parte del comité de zoning organizado por el antes mencionado Herbert Hoover, secretario del Departamento de Commerce del Gobierno norteamericano, cuyo cometido era la recolección y la organización de la información sobre el zoning con el objetivo final de redactar la Standard State Zoning Enabling Act, finalmente aprobada en 1923. Años más tarde, cuando Hoover llegó a la presidencia del Gobierno de los Estados Unidos, Kimball fue nombrada miembro del comité de investigación de la President's Conference on Home Building and Home Ownwership. Para Hoover «los objetivos morales y sociales del modo de vida americano pueden ser cumplidos sólo por una nueva concepción del city building", como se recogía en el Manual of information on city planning and zoning, including references on regional, rural, and national planning (Kimball, 1923), a él dedicado. La base epigráfica sobre la que expone la información contenida en el manual es la definida en A City-Planning Classification (Pray y Kimball, 1913). Theodora consideraba el zoning el campo «más activo dentro del city planning» (Kimball, 1921, p. 44), para el que reclamaba una legislación integral.

En la memoria de la delegación española en el congreso de 1925 de la International Federation of Housing and Town Planning en Nueva York, reseñada por Theodora en la revista City Planning (Kimball, octubre 1926, p. 309), Federico López Valencia aportaba una sintética visión del zoning norteamericano. El aspecto legisla-

12 Concepto jurídico que dota de determinadas facultades administrativas. 
tivo era prioritario, ya que las reclamaciones particulares podían convertir al zoning en un embrollo legal, tal como exponía Edward Basset, apostillaba Ford y confirmaba Joseph Stubben al manifestar que en la experiencia alemana «también tuvo el zoneamiento ${ }^{13}$ dificultades con los tribunales, hasta que sus ventajas fueron tan evidentes que se reconoció su legalidad» (López Valencia, 1926, p. 45).

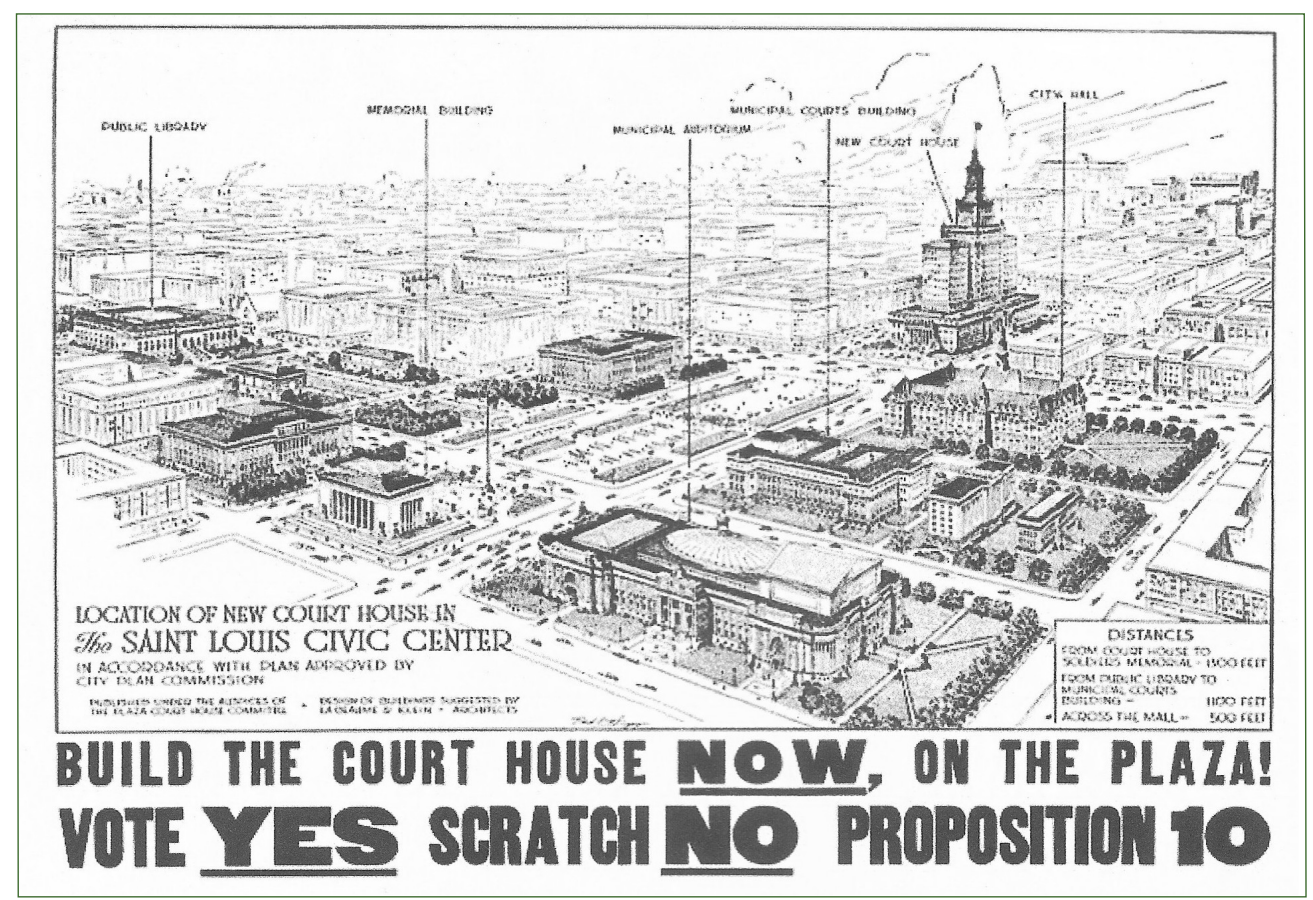

Figura 5a. Usado en la campaña para asegurarse la aprobación de la propuesta de una plaza para la instalación del edificio emblemático de St. Louis Civic Center.

Fuente: Kimball, T. y Hubbard, H. Our cities, to-day and to-morrow; a survey of planning and zoning progress in the United States. Cambridge, Mass.: Harvard University Press, 1929, p. 81.

La justificación de su éxito pudiera estar en que, en el fondo, el sistema de zoning a quien más beneficiaba era a los intereses privados de los propietarios, dado que al mantener el carácter de los barrios, protegiéndolos de invasiones no deseadas, se garantizaban los valores de las inversiones inmobiliarias. No es de extrañar por tanto que el promotor de la puesta en marcha de la primera experiencia institucionalizada del zoning norteamericano, la New York Zoning Resolution (1916), desarrollada como proyecto por George B. Ford y en su aspecto legalislativo por Edward M. Basset, fuera la poderosa Fifth Avenue Commission, entidad formada en 1907 por residentes y comerciantes del entorno para protegerse contra los desarrollos urbanos en los que las industrias y las clases inferiores se aproximaban peligrosamente y por los rascacielos que desbordaban la altura de Manhattan.

13 Zoneamiento era el vocablo utilizado inicialmente en castellano hasta la consolidación de "zonificación". 


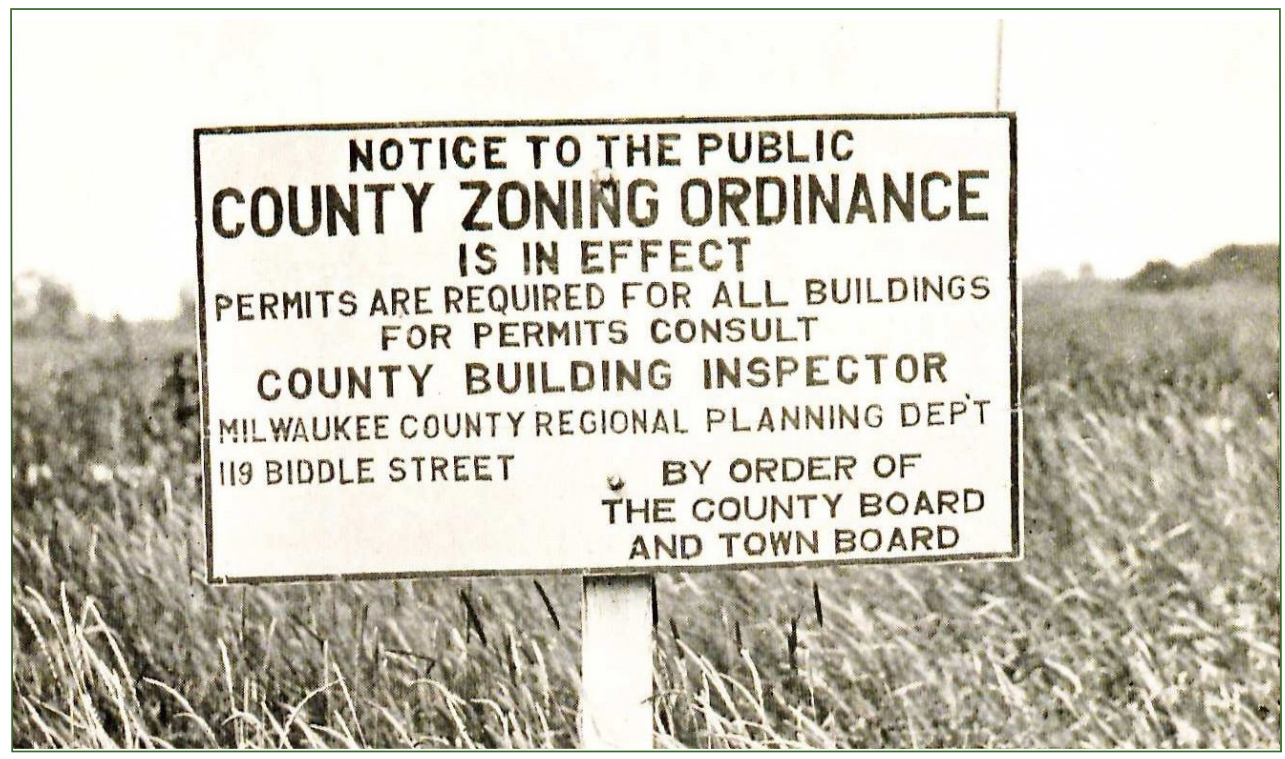

Figura 5b. El condado de Milwaukee protege sus planes.

Fuente: Kimball, T. y Hubbard, H. Our cities, to-day and to-morrow; a survey of planning and zoning progress in the United States. Cambridge, Mass.: Harvard University Press, 1929, p. 63.

\section{Del city planning al regional planning: de la ciudad-jardín europea a la ciudad en la región norteamericana}

En 1925 Vincent H. Hubbard comenzó la edición de la revista cuatrimestral City Planning, la cual se presentaba con el subtítulo Regional Planning-Rural Planning-Town Planning, concebida como el órgano oficial de difusión del American City Planning Institute y de la National Conferences on City Planning. Este matiz territorial del urbanismo americano, en base al concepto geddesiano de regional planning, no era ajeno a su imbricación con el landscape architecture. Claro exponente de ello es el nombre de Regional Planning Association of America, que Clarence Stein, Lewis Mumford y Benton Mackaye eligieron en 1923 para la primera asociación norteamericana de la ciudad-jardín.

La revista City Planning sucedía a la extinta The City Plan y fue el antecedente de la actual The Journal of the American Planning Association. "City Planning es cooperación. City Planning es el trabajo de la totalidad de la sociedad", comenzaba el primer editorial de la revista. Acompañaban a Hubbard, Charles W. Eliot, sobrino de Charles Eliot como asistente, puesto que después pasarían a desempeñar Gordom Culham y Howard Menhinick, estudiantes en formación, y un comité de editores compuesto por Edward M. Basset, Frank B, Williams, George B. Ford, Flavel Shurtleff y Theodora Kimball Hubbard. De entre los deseos de suerte para la nueva revista destacaron los de Herbert Hoover, en una carta dirigida a «Mrs. Hubbard», y los de Ebenezer Howard como presidente de la International Federation of Housing and 
Planining, con su artículo «A greeting from the International Federation». En cada uno de los números de la revista, se incluyó una sección de referencias bibliográficas y recensión de las obras coetáneas más significativas bajo la firma T. K. H., Theodora Kimball Hubbart, a los que pronto añadió la firma de Katherine MacNamara.

Para la implantación de la Harvard City Planning School en los duros momentos de recesión de la economía de finales de los años veinte, Hubbard contó con una sustanciosa beca anual, durante un periodo de siete años, de la Rockefeller Foundation. El patrocinio privado, a modo de becas o financiación de proyectos, se convirtió en el agente indispensable para la puesta en marcha de cualquier iniciativa de investigación en el ámbito americano. Las fundaciones Rockefeller, Russell Sage o Carnegie son nombres inseparables del devenir del urbanismo estadounidense.

Otro patrocinador de la Harvard City Planning School fue el neoyorkino James F. Curtis, alumno de Landscape Architecture de la promoción de 1897 y amigo de Charles Dyer Norton, uno de los responsables del Plan de Chicago de 1906 y del Plan de Nueva York de 1929, el cual facilitó fondos para la creación de la cátedra Charles Dyer Norton de Regional Planning ${ }^{14}$, que ocupó Hubbard. En 1929, los tres departamentos de la Faculty of Architecture - Regional Planning, Landscape Architecture y Architecture - se agruparon bajo la Graduate School of Design. Los planes para la nueva escuela eran del mismo carácter que los de la School of Landscape Architecture y la School of Architecture, y las tres seguían compartiendo espacio en el emblemático Robinson Hall y en el Old Fogg. Este grado de Master of City Planning fue el primero ofertado por una universidad americana. Asignaturas destacadas en los estudios eran, en el primer año, Bellas Artes, con Historia Medieval, Renacimiento y Arte Moderno; en el segundo año, Ética social, Problemas de vivienda y Aspectos sociales del urbanismo y, en el tercer año, Gobierno municipal y Administración municipal.

Fue también una beca, en este caso de la Milton Fund for Research, la que permitió sacar adelante el proyecto Our cities, to-day and to-morrow; a survey of planning and zoning progress in the United States (Hubbard y Kimball, 1929), dedicado a estudiar y analizar el progreso del planeamiento y el zoning en los Estados Unidos. Sus contemporáneos vieron el trabajo como una especie de guía "Baedeker" en city planning. Un total de 120 ciudades fueron visitadas por Howard K. Menhinick, entonces estudiante en Harvard City Planning y posteriormente profesor auxiliar tras haberse graduado en 1923 en Landscape Architecture en el Michigan State College. Menhinick elaboró la información sobre los criterios definidos previamente por los Hubbard, que fueron incluidos como apéndice de la publicación. En la obra se trataban aspectos técnicos, legales y conceptos de planeamiento que habían de ser utilizados por los profesionales del city planning que iban a salir de la recién creada Harvard City Planning School.

14 Actualmente es la Charles Dyer Norton Chair of Regional Planning and Urbanism en la Harvard Graduated School of Design. 


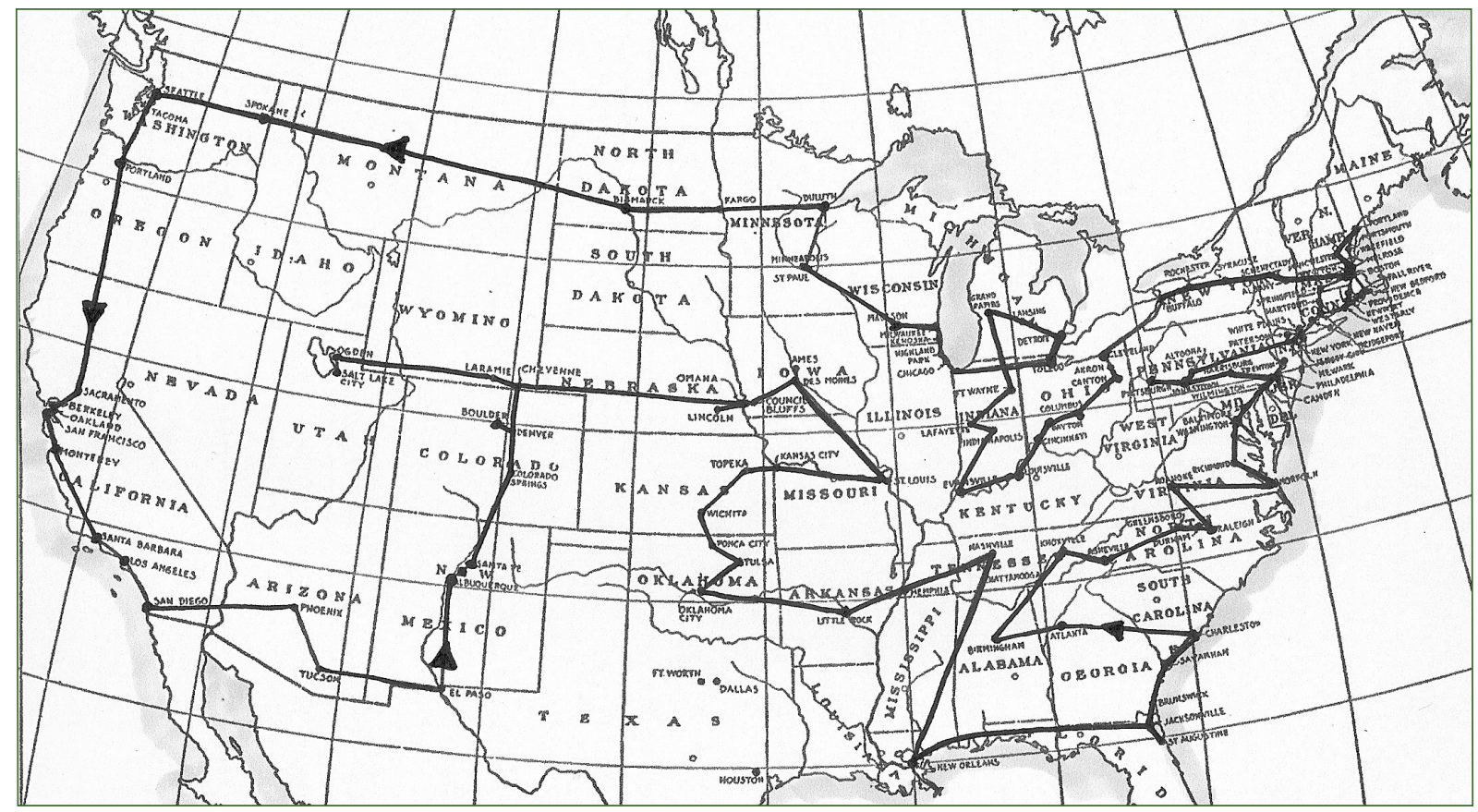

Figura 6. La ruta del estudio de campo de Harvard, 1928-1929.

Fuente: Kimball, T. y Hubbard, H. Our cities, to-day and to-morrow; a survey of planning and zoning progress in the United States. Cambridge, Mass.: Harvard University Press, 1929. p. 300

Este libro fue considerado el precursor de la serie The Harvard City Planning Studies, que incluía dos o tres publicaciones anuales con el fin de reforzar el carácter de centro de investigación con que se quería dotar a la recién creada escuela. Para los Hubbard, la investigación debía constituirse en el epicentro de la nueva escuela de city planning. En 1930 se iniciaron las publicaciones de la The Harvard City Planning Studies, de las cuales Theodora Kimball Hubbard fue la editora. Llegaron a ver la luz diez volúmenes hasta 1936. Estas obras pretendían difundir ante la comunidad قlesponsables municipales, ingenieros, arquitectos, paisajistas, consultores de planeamiento, constructores, abogados, miembros de las cámaras de comerciol los resultados de los trabajos que estaban llevando a cabo equipos de investigación sobre un aspecto concreto del urbanismo ${ }^{15}$. Los equipos de investigación estaban liderados

15 La serie The Harvard City Planning Studies estaba compuesta por los siguientes volúmenes: Volumen I. Airports. Their Location, Administration, and Legal Basis, por Henry V. Hubbard, Miller McClint, con Paul Mahoney y Howard K. Menhinick como asistentes; Volumen II. Building height, bulk, and form. How Zoning Can Be Used as a Protection against uneconomic types of buildings on high-cost land, por George B. Ford; Volumen III. Neighborhoods of small homes: economic density of low-cost housing in America and England, por Robert Harvey Whitten y Thomas Adams; Volumen IV. Urban and land uses, por Harland Bartholomew; Volumen V. Transition Zoning (1933), de Arthur C. Comey, professor asistente en la Harvard City Planning School; Volumen VI. The Design of Residential Áreas (1934), por Thomas Adams, Volumen VII. Model laws for planning cities, counties and Estates, por Edward M. Basset, Frank B. Williams, Alfred Bettam y Robert Whitten; Volumen VIII. Building Lines and Reservations for future Streets, por Russell Van Nest Black ayudado por Mary Hedges Black, Volumen IX. The steadyflow traffic system (1935), por ingeniero austriaco Fritz Malcher; y Volumen X. Biblography of planning 1928-1935, por Katherine MacNamara. 
por una personalidad de prestigio experta en la materia y un equipo de estudiantes. Las publicaciones debían despertar el debate sobre la materia tratada y así abrir nuevas líneas de investigación. Así lo explicaban los Hubbard en el prefacio del primer volumen, Airports. Their Location, Administration, and Legal Basis ${ }^{16}$.

Según se recoge en el anuario del curso 1932/33 de la Universidad de Harvard, eran seis proyectos de investigación los que se iban a llevar a cabo simultáneamente en el seno de la City Planning School. Theodora Kimball lideraba junto con Raymond F. Leonard el grupo de investigación sobre el tema «Ayudas a la publicidad en el urbanismo", en el que se involucró con limitada dedicación por su delicado estado de salud y que se mantuvo abierto hasta su eliminación por falta de financiación en el curso 1936/37, ya fallecida Theodora ${ }^{17}$. El último volumen de la colección Biblography of planning 1928-1935, por Katherine MacNamara se publicó como un homenaje póstumo a Theodora Kimball.

En 1936, año en que el City Planning pasó a denominarse formalmente Regional Planning, la Universidad de Harvard renunció a ofrecer enseñanza técnica de la materia por la falta de fondos tras la finalización de la beca de la Rockefeller Foundation. La caída de actividad mantenida hasta entonces se agudizó con la retirada de Henry V.Hubbard, que en 1941 pasó a ser catedrático emérito. La Regional Planning School no se relanzaría hasta 1945, aprovechando las posibilidades que ofrecían los trabajos de reconstrucción de la posguerra.

Ese proceso de cese de la actividad no estuvo exento de polémica. Así, resulta de especial interés la preocupación por la situación de la biblioteca del centro, como recogió el periódico de la Universidad de Harvard en 1940: «Mientras tanto, un comité investigará los modos de continuar la enseñanza o investigación en regional planning con la finalidad de dar el mayor uso a la biblioteca del departamento, considerada la más avanzada y completa del mundo, y para proveer más efectivamente al próximo tenedor de la Norton Professorship» ${ }^{18}$.

\section{Conclusión}

Para algunos estudiosos, entre ellos Heidi Hohman, la figura de Theodora Kimball no ha sido lo suficientemente valorada por la historiografía en parte porque no

16 Este volumen fue traducido al español en 1934 por el cubano Pablo Carrera Jústiz, en su calidad de aficionado a la aviación.

17 Las otras investigaciones abiertas durante ese año fueron: The influence of Parkways on Land Values and Social Values, liderada por John Nolen; Model up-to-date Zoning Law and Planning Law for Use in the United States, liderada por Fran B. Williams, Edward M. Basset, Robert Whitten y Alfred Bettman; The Establishment and Protection of Mapped Streets and Building Lines, con Russell Van Nest Black; y Methods of Preserving Open Development Áreas, con Arthur C. Comey y Frank B. Williams

18 School of Design To Drop Regional Planning Courses. The Harvard Crimson, 16 de marzo de 1940. 
produjo proyectos construidos. Peter Hall seleccionó sus obras Manual of information on city planning and zoning, including references on regional, rural, and national planning y Our cities, to-day and to-morrow; a survey of planning and zoning progress in the United States como la principal contribución de los Hubbard al urbanismo, siendo también citado el último trabajo antes referido en el American city planning from 1890 de Mel Scott. Para Eugene L. Birch, Theodora Kimball, constituye una de las aportaciones intelectuales más representativas desde «la tradición no profesional del urbanismo». Iniciativas como el Premio Theodora Kimball Hubbard de la Society for American City and Regional Planning History prueban su puesta en valor por parte de la cultura norteamericana.

Theodora Kimball fue editora, crítica e investigadora, aunque no fue propiamente una creadora, ni aportó nuevos conceptos al urbanismo. Sin embargo, su amplio trabajo fue un instrumento fundamental para entender los procesos formativos del urbanismo norteamericano, integrando el landscape architecture, zoning, city planning, y regional planning. Asumió un activo papel en el afianzamiento progresivo de los contenidos del urbanismo, contribuyendo así a la formación del campo teórico desde la literatura técnica. Su concienzudo trabajo intelectual como camino para acceder al conocimiento de modo que permitiera la creación de un lenguaje común como herramienta de trabajo, fue un esfuerzo notable que merece la pena valorar.

En el nacimiento de una disciplina, una de las primeras necesidades que identifican la demarcación de su campo de conocimiento es la creación de un lenguaje específico propio. La expresión de nuevos conceptos mediante una jerga técnica que los clasifique, integre y permita transmitir con precisión es clave. En el caso del urbanismo, se emplean numerosos neologismos que abren constantemente nuevos caminos que permitan denominar una realidad que ya no es la de la ciudad histórica, tal y como señaló en su día Ignasi de Solà-Morales (Solà-Morales, 2002, p. 24).

El urbanismo como ámbito de conocimiento se genera a modo de redes temporales y espaciales de tan distinta naturaleza, teórica y material, social y cultural, que ha de ser debidamente encauzado, racionalizado y sistematizado para evitar la transformación de esa red de conocimientos en una maraña indescifrable de información. Desde estas ideas es donde cabe valorar la singular y valiosa aportación de Theodora Kimball a la construcción de la ciudad moderna.

\section{Bibliografía}

AA. VV. La ciudad americana, Barcelona: Gustavo Gili, 1975.

AMERICAN INSTITUTE OF ARCHITECT COMMITTEE ON TOWN PLANNING. City planning progress in the United Estates, Washington, George Burdett Ford Ed., The Journal of the American Institute of Architects, 1917.

AMERICAN SOCIETY OF LANDSCAPE ARCHITECTS. Henry Vincent Hubbard. An official minute on his professional life and work. Landscape Architecture, 2, enero, 
1948, p. 49.

BIRCH, E. From Civic Work to City Planner, Women and Planning, 1890 to 1980, en Krueckeberg, D. The American Planner. Nuevo Brunswick: Center for Urban Policy Research, 1994, pp. 469-506.

CARRERA JÚSTIZ, F. El Urbanismo en los Estados Unidos: Theodora Kimball. Revista Municipal y de Intereses Económicos, XVI (9) abril, 1921.

CORT, C. La Conferencia Interaliada de Urbanismo. Boletín de la Sociedad Central de Arquitectos, (63), 3, (64), 3-6, (65), 3-7, (66), 4-6, 1919.

DONNELLY, J. Theodora Kimball Hubbard 1887-1935. Simmons Review, enero, 1936, pp. 44-45.

FORD, G. L'Urbanisme in practique. París: Ernest Leroux, 1920.

GARCÍA MERCADAL, F. Bibliografía de Urbanismo. Arquitectura, noviembre, (112), 1928, p. 202.

GEDDES, P. Cities in evolution, Londres: Williams \& Norgate, 1915.

HALL, P. Cities of tomorrow. Oxford: Blackwell Publishers, 1997.

HARRIS, S. Blue pencils and hidden hands. Nueva York: Northeastern University Press, 2004.

HOHMANN, H. Theodora Kimball Hubbard and the "Intellectualization" of Landscape Architecture, 191 1-1935. Landscape Journal, 25 (2), septiembre, 2006, pp. 169-186. DOI: 10.3368/lj.25.2.169

HUBBARD, H. \& KIMBALL, T. The Arrangement of the Professional Collections of a Landscape Architect. Landscape Architecture, (3), enero, 1913, pp. 76-89.

HUBBARD, H. \& KIMBALL, T. An introduction to the study of landscape design. Nueva York: Macmillan Company, 1917

HUBBARD, H. \& KIMBALL, T. Landscape architecture ; a comprehensive classification scheme for books, plans, photographs, notes and other collected material, with combined alphabetic topic index and list of subject headings. Cambridge, Mass.: Harvard University Press, 1920.

HUBBARD, H. \& KIMBALL, T. Our cities, to-day and to-morrow; a survey of planning and zoning progress in the United States. Cambridge, Mass.: Harvard University Press, 1929.

KIMBALL, T. A brief survey of recent city planning reports in the United States. Landscape Architecture, 1912, pp. 111-126.

KIMBALL, T. Classified selected list on references on City Planning. Boston: National Conference on City Planning. Publicada la separata Streets: Their Arrangement, Lighting and Planning. Special Libraries, 6 (3), 1915, pp. 43-48.

KIMBALL, T. Personality and Human Beings in Town-Planning Work. American City, 22, junio, 1920, p. 609.

KIMBALL, T. (Ed.). Municipal accomplishment in city planning and published city plan reports in the United States. Boston: National Conference on City Planning, 1920.

KIMBALL, T. Town planning courses at the School of Landscape Architecture, 
Harvard University. Journal of Town Planning Institute of Canada, ( 1 ) febrero, 1921 , pp. $11-12$.

KIMBALL, T. Brief review of city planning in the United States. National Municipal Review, enero 1921, pp. 39-50. Serie anual publicada desde 1917 a 1926.

KIMBALL, T. A review of city planning in the United States, 1920-1921. Town Planning Review, 9 (4), mayo, 1922.

KIMBALL, T. Manual of information on city planning and zoning, including references on regional, rural, and national planning. Cambridge, Mass.: Harvard University Press, 1923.

KIMBALL, T. H.W. S. Cleveland. An American pioneer in Landscape Architecture and City Panning. Landscape Architecture, (20) enero, 1930, pp. 92-111.

KIMBALL, T. Planning information up-to-date; a supplement, 1923-1928, to Kimball's Manual of information on city planning and zoning, including references on regional, rural, and national planning. Cambridge, Mass.: Harvard University Press, 1928.

KNACK, R. \& MECK, S. \& STOLLMAN,I. The real story behind the standard planning and zoning acts in the 20s. Land use Law \& Zoning Digest, febrero 1996, pp. 3-9.

KRUECKEBERG, D. The Story of the Planner's Journal, 1915-1980. Journal of the American Planning Association, 46 (1), 2007, pp. 5-21.

LANDSCAPE ARCHITECTURE. Theodora Kimball Hubbard: A Biographical Minute, 26 (2) enero, 1936, pp. 53-55.

LÓPEZ VALENCIA, F. Congreso Internacional de Trazado de Poblaciones (Nueva York, 1925). Memoria. Madrid: Instituto de Reformas Sociales, 1926.

MEYER, H. Check list of references on City Planning. Special Libraries. Indianapolis: Special Libraries Association, 3 (5) mayo, 1912, pp. 61-123.

NOLEN, J. Some Random impressions in looking over twenty volumes of Landscape Architecture. Landscape Architecture, 20 (4) julio, 1930, p. 296.

OLMSTED, F. \& KIMBALL, T. (Eds.). Frederick Law Olmsted, landscape architect, 1822-1903. Nueva York: G. T. Putnam's Sons, 1922.

OLMSTED, F. \& KIMBALL, T. Central Park as a work of art and as a great municipal enterprise, 1853-1895, Nueva York: G. P. Putnam's Sons, 1928

POWER, G. The advent of zoning. Planning Perspectives, 4 ( 1) 1989, pp. 1-13.

DOI: $10.1080 / 02665438908725670$

SCOTT, M. American City Planning since 1890. Berkeley: University of California Press, $\quad$ 1995, $1^{\text {a }}$ ed. 1969.

SOLÀ-MORALES, I. Territorios. Barcelona: Gustavo Gili, 2002.

STURGIS, J. \& KIMBALL, T. A city planning classification, preliminary outline. Cambridge: Harvard University Press, 1913.

STURGIS, J. \& KIMBALL, T. City planning; a comprehensive analysis of the subject arranged for the classification of books, plans, photographs, notes and other collected material, with alphabetic subject index. Cambridge: Harvard University Press, 1913. 
(C) Copyright: María Cristina García González y Salvador Guerrero, 2017

(c) Copyright Scripta Nova, 2017.

Ficha bibliográfica:

GARCíA GONZÁLEZ, María Cristina; GUERRERO, Salvador. El urbanismo como acto de comunicación: Theodora Kimball Hubbard y la Biblioteca de la Harvard Landscape Architecture School. Scripta Nova. Revista Electrónica de Geografía y Ciencias Sociales. [En línea]. Barcelona: Universidad de Barcelona, 1 de febrero de 2017, vol. XXI, no 555. <http://revistes.ub.edu/index.php/ScriptaNova/article/view/555>.

ISSN: 1138-9788. 